\title{
Antibody biosensors for the measurement and characterization of soluble CD147 molecules
}

\author{
Witida Laopajon, ${ }^{1,2}$ Nuchira Takheaw, ${ }^{1}$ Saichit Khummuang, ${ }^{1}$ Tanyaluck Kampoun, ${ }^{1}$ Kantinan Cheunsirikulchai, ${ }^{1}$ \\ Watchara Kasinrerk, ${ }^{1,2}$ Supansa Pata ${ }^{1,2}$
}

\begin{abstract}
Background: Soluble CD147 (sCD147) is the shed form of membrane-bound CD147, which is involved in the regulation of cellular functions. The presence of sCD147 in body fluids is associated with several diseases.

Objective: In this study, we aimed to establish antibody $(\mathrm{Ab})$ biosensors for the simultaneous differential detection of the general and truncated forms of sCD147.

Method: By combining biolayer interferometry technology (BLItz) and different anti-CD147 monoclonal antibodies (mAbs) specific to different extracellular domains of the CD147 molecule, Ab-based biosensors were established to rapidly measure and characterize sCD147 isoforms.

Results: Two types of Ab-biosensors, desginated the single Ab-biosensor and double Ab-biosensor, were established for the measurment and characterization of sCD147 isoforms. For the single Ab-biosensor system, monoclonal antibodies specific for CD147 domain 1 (D1) or domain 2 (D2) were immobilized on the sensor tips and used for the quantification of sCD147 using a BLItz optical interferometric biosensor. For the double Ab-biosensor system, following the single Ab-biosensor step, secondary anti-CD147 mAbs specific for each domain of the CD147 molecule were added and monitored by a BLItz biosensor. By combining the results obtained from the single Ab- and double Ab-biosensors, sCD147 isoforms including the general form (D1 linked to D2) and the truncated forms (sCD147 containing D1 or D2) could be determined.
\end{abstract}

Conclusions: This method may be a beneficial tool for the determination of sCD147 isoforms for disease diagnosis and prognosis as well as for the definition of the cellular mechanisms of the immune system.

Keywords: CD147, soluble CD147, isoform, biosensor, monoclonal antibody

\footnotetext{
From:

${ }^{1}$ Division of Clinical Immunology, Department of Medical Technology, Faculty of Associated Medical Sciences, Chiang Mai University, Chiang Mai 50200, Thailand

${ }^{2}$ Biomedical Technology Research Center, National Center for Genetic Engineering and Biotechnology, National Science and Technology Development Agency at the Faculty of Associated Medical Sciences, Chiang Mai University, Chiang Mai 50200, Thailand
}

\section{Introduction}

CD147, also called M6 antigen, ${ }^{1}$ basigin, ${ }^{2}$ or extracellular matrix metalloproteinase inducer (EMMPRIN), ${ }^{3}$ is a $50-60 \mathrm{kDa}$ type I integral transmembrane glycoprotein. This molecule is broadly expressed by many cell types, including epithelial cells, endothelial cells, leukocytes, red blood cells and platelets. ${ }^{4}$ The CD147 molecule contains immunoglobulin (Ig)-like domains in the extracellular part with a single transmembrane domain and a short cytoplasmic tail. ${ }^{1-7}$ Due to alternative splicing,

\section{Corresponding author:}

Supansa Pata

Division of Clinical Immunology, Department of Medical Technology, Faculty of Associated Medical Sciences, Chiang Mai University, Chiang Mai 50200, Thailand.

E-mail: supansa_pata@hotmail.com

CD147 has been categorized into four isoforms (basigin-1 to -4 ), which differ in the number of Ig-like domains in the extracellular region. ${ }^{5}$ Basigin-2 or the general form is the major form containing two Ig-like domains (membrane-distal domain; D1 and membrane-proximal domain; D2). Basigin-1 is a retina-specific form and has three extracellular Ig-like domains (D0, D1 and D2). Basigin-3 and basigin-4 have only one extracellular domain (D2) ${ }^{5,6} \mathrm{CD} 147$ has been characterized 
as a multifunctional molecule, which is involved in many cellular processes including cell adhesion, cell migration and $\mathrm{T}$ cell activation. ${ }^{7-10}$ In addition, CD147 is able to induce matrix metalloproteinase (MMP) production, which is associated with cancer progression and metastasis. ${ }^{11,12}$ Elevated CD147 expression has been reported in various cancers. ${ }^{13-15}$

Over the past decade, soluble CD147 (sCD147) has been uncovered in addition to membrane-bound CD147. sCD147 production is mediated by two different mechanisms: microvesicular secretion and proteolytic cleavage. ${ }^{16,17}$ Full length sCD147 is released by microvesicular secretion, ${ }^{16,18}$ whereas the extracellular fragment of sCD147 is shed either in the general form (D1 linked to D2) or only in the D1 form via MMP-dependent cleavage. ${ }^{17,19}$ The two extracellular Ig-like domains of the CD147 molecule contain three distinct N-glycosylation sites: one asparagine on D1 and two asparagines on D2.,20 The different glycosylation sites on D1 and D2 result in its different functions and binding capacity. The binding of CD147 D1 and its ligands contributes to some functions of the CD147 molecule, including MMP induction, cell adhesion and migration. ${ }^{5,12,21}$ The D2 region of the CD147 molecule is responsible for the association with integrin and annexin II, which are involved in cell adhesion, invasion and migration. ${ }^{21}$ An interaction between D2 and caveolin-1 has been demonstrated, and this interaction suppresses MMP production. $^{21,22}$

The different shed sCD147 isoforms might initiate different cellular responses, which may be involved in immunoregulation and the mechanisms underlying disease. The presence of sCD147 in clinical samples has been reported in many diseases such as systemic sclerosis, hepatocellular carcinoma, ankylosing spondylitis and coronary diseases. ${ }^{12,23-26}$ Detection of sCD147 forms in clinical samples may, therefore, be a useful marker for the diagnosis and prognosis of disease. ${ }^{23,26}$ However, the currently available methods for measuring sCD147 are unable to detect and characterize the truncated form of sCD147.23,27,28 The structure of shed sCD147 in clinical samples still not well -characterized. ${ }^{17-19,29}$ In the present study, a novel biosensor system was developed. Using a combination of biolayer interferometry technology (BLItz) and different anti-CD147 monoclonal antibodies (mAbs) specific to different domains of the CD147 molecule, Ab based-biosensors were established to simultaneously and rapidly measure and characterize sCD147 isoforms.

\section{Methods \\ Antibodies and reagents}

The anti-CD147 mAbs recognizing D1 of CD147 molecule (CD147 D1) clone M6-1E9 (isotype IgG2a) and the anti-CD4 $\mathrm{mAb}$ clone CD4-COS1 (IgG2a) were produced in our laboratory. ${ }^{9,30}$ The anti-CD147 mAb binding CD147 D1 clone MEM-M6/3 (IgG1) and the anti-CD147 mAb specific for CD147 D2 clone MEM-M6/6 were purchased from Exbio (Prague, Czech Republic). ${ }^{31}$ The anti-CD3 mAb clone OKT3 (IgG1) (Ortho Pharmaceuticals, Raritan, NJ, USA), anti -CD31 mAb clone JC70A (IgG1) (Dako, Glostrup, Denmark), horseradish peroxidase (HRP) conjugated rabbit anti-mouse immunoglobulin Abs (Dako), AlexaFluor 488-anti-mouse
IgG Abs (Invitrogen, Carlsbad, CA, USA), lipofectamine 2000 (Invitrogen), EZ-Link ${ }^{\text {Tm }}$ Sulfo-NHS-Biotin (Pierce, Rockford, IL, USA) used in this study were sourced as noted. CHO-S-SFM II and DMEM medium were purchased from Gibco (Grand Island, NY, USA). The BLItz biolayer interferometry biosensor and the streptavidin sensor were obtained from FortéBio (Pall Life Sciences, Menlo Park, CA, USA).

\section{Preparation of recombinant proteins}

Chinese hamster ovarian $(\mathrm{CHO})$ cells carrying the gene encoding for the CD147 extracellular domain-human IgG Fc fusion protein (CD147Rg; contain D1 linked to D2) and CD31 extracellular domain-human IgG Fc fusion protein (CD31Rg) were kindly provided by Prof. Dr. Hannes Stockinger (Medical University of Vienna, Austria). ${ }^{31,32}$ Recombinant soluble CD147Rg and CD31Rg were prepared from the described $\mathrm{CHO}$ cells. In brief, $\mathrm{CHO}$ cells carrying the CD147Rg or CD31Rg gene were cultured in CHO-S-SFM II medium (Gibco) with $5 \mathrm{mM}$ methotrexate at $37^{\circ} \mathrm{C}$ and $5 \% \mathrm{CO}_{2}$. The culture supernatant containing CD147Rg or CD31Rg was collected and subjected to purification by affinity chromatography on a HiTrap Protein G HP column (GE Healthcare, Uppsala, Sweden), based on the human IgG-Fc part. The purified recombinant proteins were confirmed by ELISA using corresponding specific mAbs. The validated purified recombinant proteins were used as sCD147 general form (D1 linked to D2) and sCD31 (recombinant protein control).

The recombinant CD147 D1-human IgG Fc fusion protein (CD147-D1Rg) and CD147 D2-human IgG Fc fusion protein (CD147-D2Rg) were prepared using the COS7 cell expression system. COS7 cells were maintained in a DMEM medium (Gibco) supplemented with 10\% fetal bovine serum (Gibco), $100 \mathrm{U} / \mathrm{mL}$ gentamicin, $2 \mathrm{mg} / \mathrm{mL}$ Fungizone, at $37^{\circ} \mathrm{C}$ and $5 \%$ $\mathrm{CO}_{2}$. The COS7 cells $\left(5.0 \times 10^{5}\right)$ were transfected with $4 \mu \mathrm{g}$ of the pCDM8 plasmid vector encoding CD147-D1Rg or CD147-D2Rg (provided by Prof. Dr. Hannes Stockinger), using Lipofectamine 2000 (Invitrogen). The schematic illustration of CD147 molecule and plasmid vector is shown in Figure S2-A. The transfected COS7 cells were cultured in CHO-S -SFM II medium (Gibco) at $37^{\circ} \mathrm{C}$ and $5 \% \mathrm{CO}_{2} \cdot{ }^{31}$ Intracellular immunofluorescence staining was performed to determine the production of CD147-D1Rg or CD147-D2Rg. The culture supernatants were collected and used as sCD147 D1 and sCD147 D2 for the validation of the biosensor system.

\section{Determination of produced recombinant proteins by ELISA}

The purified recombinant proteins $(5 \mu \mathrm{g} / \mathrm{mL})$ were prepared in a carbonate-bicarbonate coating buffer $(\mathrm{pH} 9.6)$ and coated on the well surface of the plate at $4^{\circ} \mathrm{C}$ overnight. The free surface of each well was blocked with $2 \%$ skimmed milk in PBS. Anti-CD147 $\mathrm{mAb}$ and anti-CD31 mAb $(10 \mu \mathrm{g} / \mathrm{ml})$ were added into the wells and incubated at $37^{\circ} \mathrm{C}$ for $1 \mathrm{~h}$. After washing four times, HRP-conjugated rabbit anti-mouse immunoglobulin $\mathrm{Ab}$ was added into all wells. After incubation at $37^{\circ} \mathrm{C}$ for $1 \mathrm{~h}$, the plate was washed four times and the reactions were observed by adding a TMB substrate (Zymed) and measuring the optical density (OD) at $450 \mathrm{~nm}$. 


\section{Intracellular immunofluorescence staining}

Transfected COS7 cells were washed with PBS and fixed with $2 \%$ paraformaldehyde for 30 min on ice and permeabilized with $90 \%$ methanol for $1 \mathrm{~h}$ on ice. After washing, the cells were pre-incubated with $10 \%$ fetal bovine serum at $4^{\circ} \mathrm{C}$ for $30 \mathrm{~min}$ to block nonspecific Fc-receptor-mediated binding of mAbs. The blocked cells were then incubated for $30 \mathrm{~min}$ at $4^{\circ} \mathrm{C}$ with specific $\mathrm{mAbs}$, followed by incubation with Alexa fluor 488-anti-mouse IgG Abs (Invitrogen) for $30 \mathrm{~min}$ at $4^{\circ} \mathrm{C}$. The stained cells were analyzed under a fluorescent microscope (Nikon, Tokyo, Japan).

\section{Immobilization of biotinylated antibodies onto the streptavi- din sensor}

Anti-CD147 mAbs clones M6-1E9, MEM-M6/3 and MEM -M6/6, as well as isotype-matched control mAbs clones OKT3 and CD4-COS1, were labeled with biotin using EZ-Link ${ }^{\mathrm{mm}}$ Sulfo-NHS-Biotin (Pierce) according to the manufacturer's instructions. The biotinylated antibodies $(100 \mu \mathrm{g} / \mathrm{mL})$ were prepared in sample diluent buffer (PBS containing $0.1 \%$ bovine serum albumin, 0.02\% Tween-20, 0.05\% $\mathrm{NaN}_{3}$ ). Next, biotinylated antibodies were immobilized onto streptavidin sensors (FortéBio), which were hydrated for $15 \mathrm{~min}$ before being used. The binding of the biotinylated antibodies onto the sensors was measured using a BLItz biolayer interferometry biosensor (FortéBio) for $2 \mathrm{~min}$ to observe the association signal. The immobilized sensors were placed into the sample diluent buffer for $30 \mathrm{~s}$ to observe the dissociation of the mAbs.

\section{Determination of sCD147 using the single Ab-biosensor detec- tion system}

The mAb-immobilized sensor tips were placed into the sample diluent buffer (PBS containing $0.1 \%$ bovine serum albumin, $0.02 \%$ Tween-20, $0.05 \% \mathrm{NaN} 3$ ) for $30 \mathrm{~s}$ to set the binding baseline. The sensors were then placed into samples containing the sCD147 general form, sCD147 D1, sCD147 $\mathrm{D} 2$ or sCD31 for $60 \mathrm{~s}$ to determine the antigen-antibody association. Finally, the sensor was placed in sample diluent buffer for $30 \mathrm{~s}$ to observe antigen-antibody dissociation. The association and the dissociation signals were measured using the BLItz biolayer interferometry biosensor (FortéBio).

\section{Determination of sCD147 using the double Ab-biosensor de- tection system}

Following the sCD147 binding step of the single Ab-biosensor detection system, excess sCD147 was removed by placing the sensor tips in sample diluent for $30 \mathrm{~s}$. Next, the secondary anti-CD147 mAbs, which bind to different extracellular domains of CD147 then the primary antibody, were added. The antigen-antibody association signal was observed for $60 \mathrm{~s}$ using the BLItz biolayer interferometry biosensor (FortéBio). The sensors were then placed in sample diluent for $30 \mathrm{~s}$ to observe antigen-antibody dissociation.

\section{Data analysis}

The binding signals obtained from the biosensors were calculated as a delta binding signal, which was obtained by subtracting the binding signal at the starting point $\left(\mathrm{T}_{\mathrm{S}}\right)$ from the binding signal at the endpoint $\left(\mathrm{T}_{\mathrm{E}}\right)$, as given in the equation below. For the single Ab-biosensor system using the time point (seconds) of the antigen association step:

$$
\text { Delta binding signal }=\mathrm{T}_{\mathrm{E}} 90-\mathrm{T}_{\mathrm{S}} 30
$$

For the double Ab-biosensor system using the time point (seconds) of the second $\mathrm{mAb}$ association step:

Delta binding signal $=\mathrm{T}_{\mathrm{E}} 180-\mathrm{T}_{\mathrm{S}} 120$

In the calibration curve measurement, the limit of detection (LOD) was calculated using the following equation:

LOD signal $=$ average of delta binding signal of control + 3SD of control

The calculated LOD signals were used as the Y-value in the equation, as indicated on each calibration curve, and then the $\mathrm{X}$-value was calculated as the LOD.

\section{Results}

\section{Production of recombinant sCD147}

In order to establish a novel antibody-based biosensor for assessing sCD147 isoforms, recombinant sCD147 and other control proteins were required for use as standard materials in the process of developing the method. The sCD147 general form (D1 linked to D2), sCD147 with D1 only (CD147 D1), sCD147 with D2 only (CD147 D2) and sCD31 (as control) were produced using the mammalian expression system. The produced recombinant proteins were validated for their characteristics, as previously described, ${ }^{31,32}$ prior to use. The produced materials had the correct properties (Figures $\mathbf{S 1}$ and S2 in the Supplementary materials section). These recombinant proteins, therefore, could be used in the development of the Ab-biosensor.

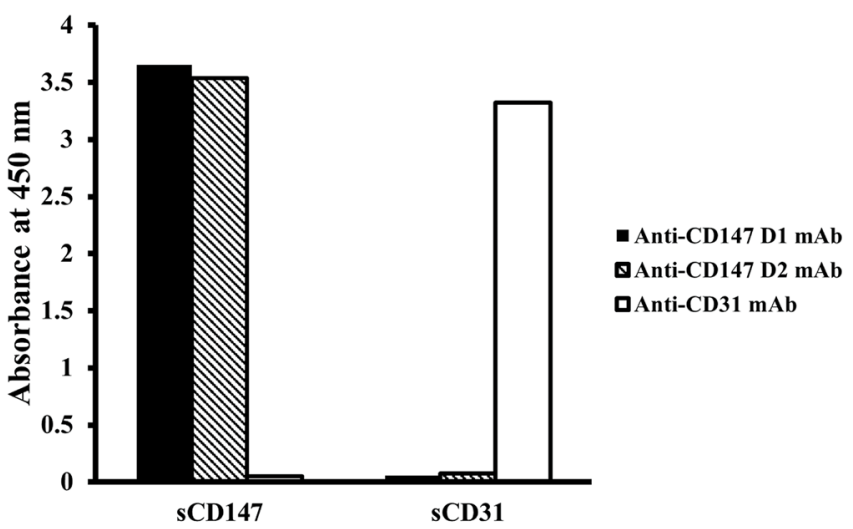

Figure S1. Validation of the produced sCD147 general form by ELISA. Purified recombinant sCD147 general form and sCD31proteins were coated on an ELISA plate. The ELISA test was performed using anti-CD147 mAb clone MEM-M6/3 (CD147-D1 specific), anti-CD147 mAb clones MEM-M6/6 (CD147-D2 specific) and anti-CD31 mAb clone JC70A (CD31 specific). 
A
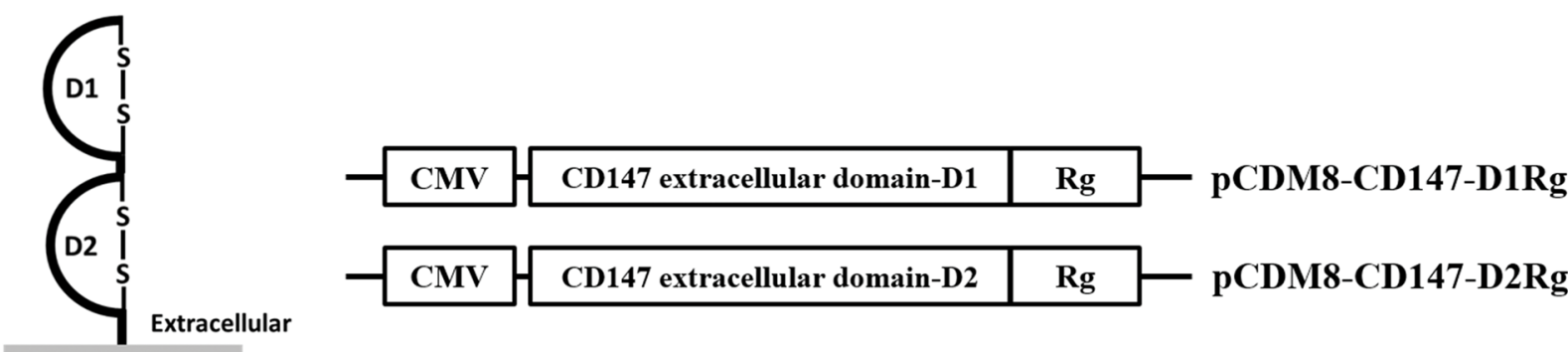

Plasma membrane

Cytoplasm

Structure of CD147

B

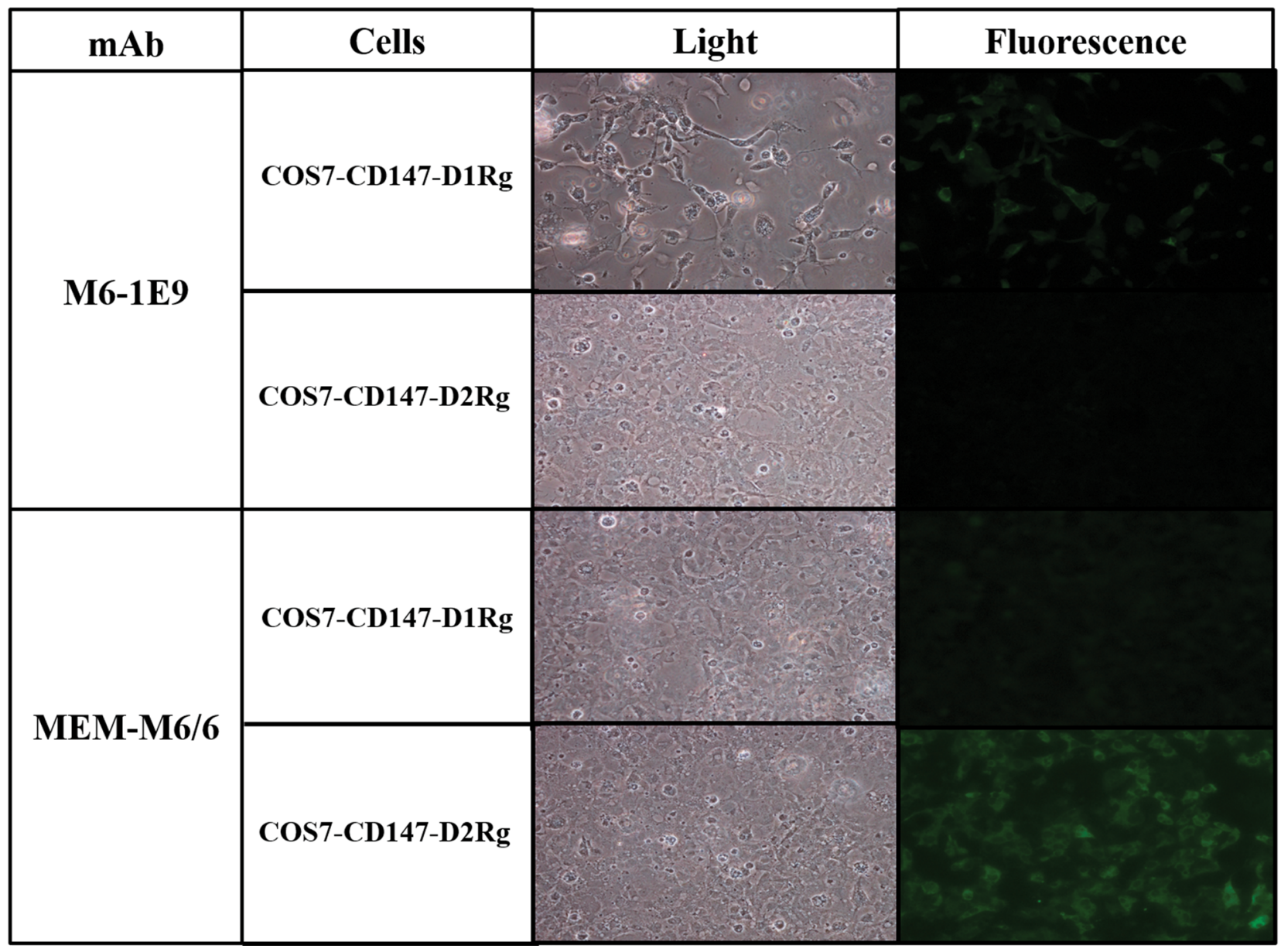

Figure S2. Validation of the produced sCD147 D1 and sCD147 D2 isoforms by immunofluorescence analysis. (A) Schematic illustration of CD147 molecule (general isoform; Basigin-2) and the plasmid vectors for the production of CD147- D1Rg and CD147D2Rg. (B) COS7 cells were transfected with plasmid encoding CD147 D1 (CD147-D1Rg) or CD147 D2 (CD147-D2Rg). Intracellular immunofluorescent staining was performed using anti-CD147 mAb clone M6-1E9 (CD147 D1 specific) and anti-CD147 mAb clone MEM-M6/6 (CD147 D2 specific). Light and fluorescent imaging are demonstrated.

\section{Immobilization of mAbs onto the biosensor tip}

In order to develop Ab-biosensors, biotin was first labeled on anti-CD147 mAbs, 2 mAbs specific for CD147 D1 and $1 \mathrm{mAb}$ specific for CD147 D2 as well as isotype-matched control mAbs. The labeled mAbs were then immobilized on the streptavidin sensor tips. As demonstrated in Figure 1, all the biotinylated
mAbs showed binding signals with streptavidin sensors when compared to the non-biotinylated mAbs. These data suggest that all mAbs were successfully labeled with biotin and could bind onto the streptavidin sensor tips. 


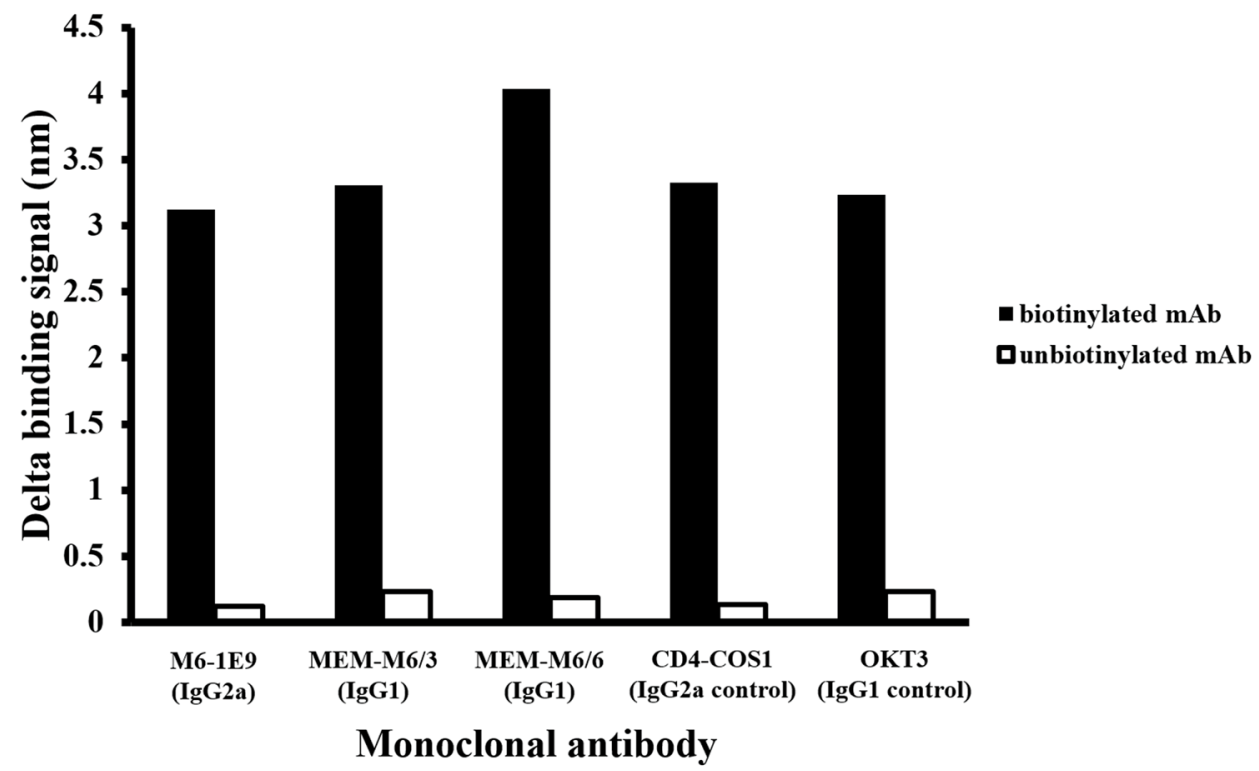

Figure 1. Binding of biotinylated monoclonal antibodies onto streptavidin sensor tip. Anti-CD147 mAbs clones M6-1E9 and MEM-M6/3 (CD147 D1 specific), anti-CD147 mAb clone MEM-M6/6 (CD147 D2 specific), anti-CD4 mAb clone CD4-COS1 (CD4 specific), and anti-CD3 mAb clone OKT3 (CD3 specific) were labeled with biotin. The biotinylated mAbs or unbiotinylated mAbs (as indicated) were immobilized onto streptavidin sensors. The binding of the mAbs onto the sensors was measured using a BLItz optical interferometric biosensor. The isotypes of the mAbs were indicated in the parenthesis.

\section{Single Ab-biosensor system for the determination of soluble CD147}

In order to determine the different forms of sCD147, two types of Ab-biosensors, namely single Ab-biosensor and double Ab-biosensor, were designed. For the single Ab-biosensor, biotinylated anti-CD147 mAbs specific for CD147 D1 or CD147 D2 were coated on sensor tips and used for the quantitation of sCD147 using BLItz optical interferometric biosensor in real-time. The anti-CD147 D1 mAbs clone M6 -1E9 and MEM-M6/3, specifically, bound to the recombinant sCD147 D1 but not to sCD147 D2. On the other hand, the anti-CD147 D2 mAb clone MEM-M6/6 recognized only sCD147 D2. Very low binding signals of both the sCD147 D1 and the sCD147 D2 molecules were observed when using

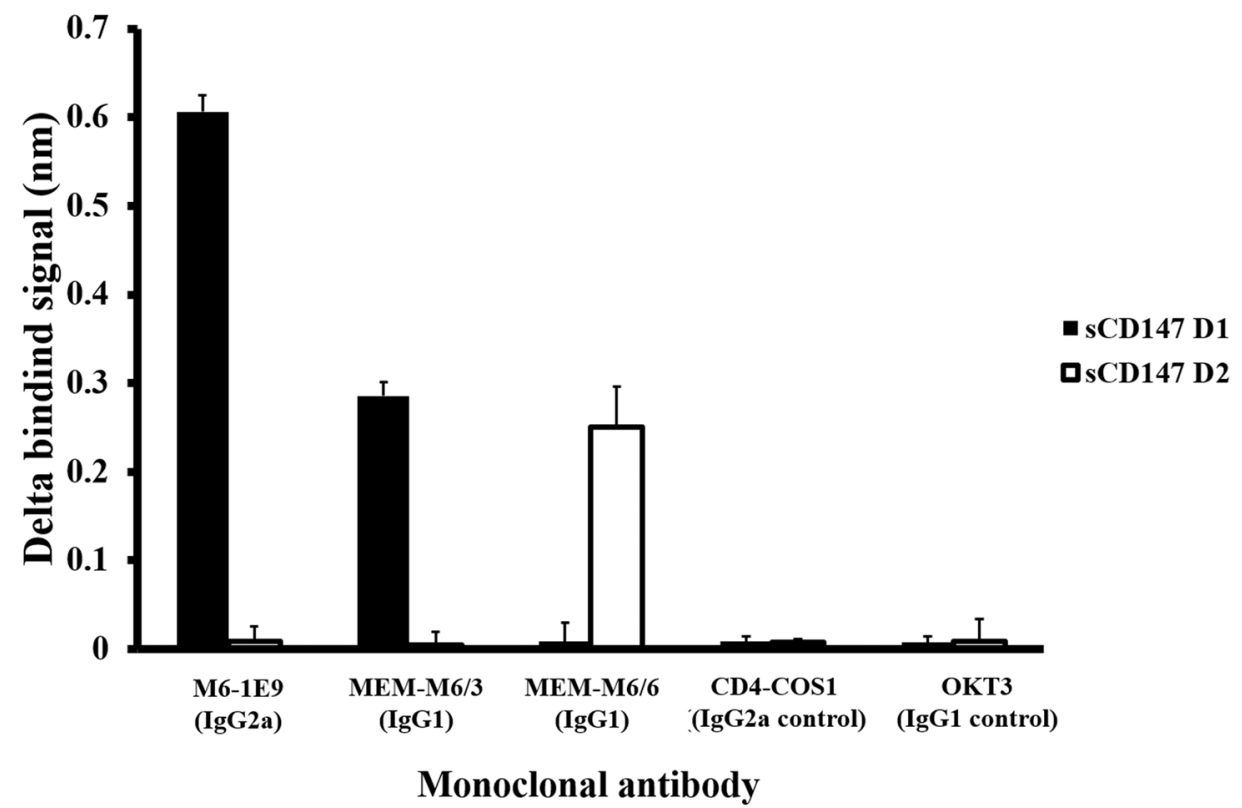

Figure 2. Specificity of anti-CD147 mAb-biosensors. Anti-CD147 mAbs clones M6-1E9 and MEM-M6/3 (CD147 D1 specific), anti-CD147 mAb clone MEM-M6/6 (CD147 D2 specific), and isotype matched control mAbs (anti-CD4 mAb clone CD4-COS1, anti-CD3 mAb clone OKT3) were immobilized on sensors. The mAb-biosensors were used to determine their specificity by using recombinant CD147 D1 and CD147 D2. The binding signals were determined using a BLItz biosensor and plotted as the mean \pm SD of three independent experiments. The isotypes of the mAbs were indicated in the parenthesis. 
isotype matched control mAb-coated sensors (Figure 2). The results illustrate that the anti-CD147 mAb coated sensors have specific recognition of their target molecules. Therefore, the established Ab-biosensor system could be used for the measurement of sCD147.

The developed single Ab-biosensors were then validated for their detection of sCD147. Both anti-CD147 D1 specific and anti-CD147 D2 specific mAbs demonstrated the binding signal with the sCD147 general form (containing D1 linked to D2) but showed a negative signal with the control antigen, sCD31 protein (Figure 3A). The limit of detection (LOD) for the detection of sCD147 by each anti-CD147 mAb was determined. LODs at concentrations of $0.89 \mu \mathrm{g} / \mathrm{mL}$ and $1.132 \mu \mathrm{g} / \mathrm{mL}$ were obtained using anti-CD147 D1 mAb clones M6-1E9 and MEM-M6/3, respectively (Figures 3B and C). LOD was obtained at a concentration of $8.137 \mu \mathrm{g} / \mathrm{mL}$ using $\mathrm{mAb}$ MEM-M6/6 (Figure 3D). The results indicate that single Ab-biosensor systems were successfully developed for the detection of sCD147 with a detection limit in the nanogram range.

A

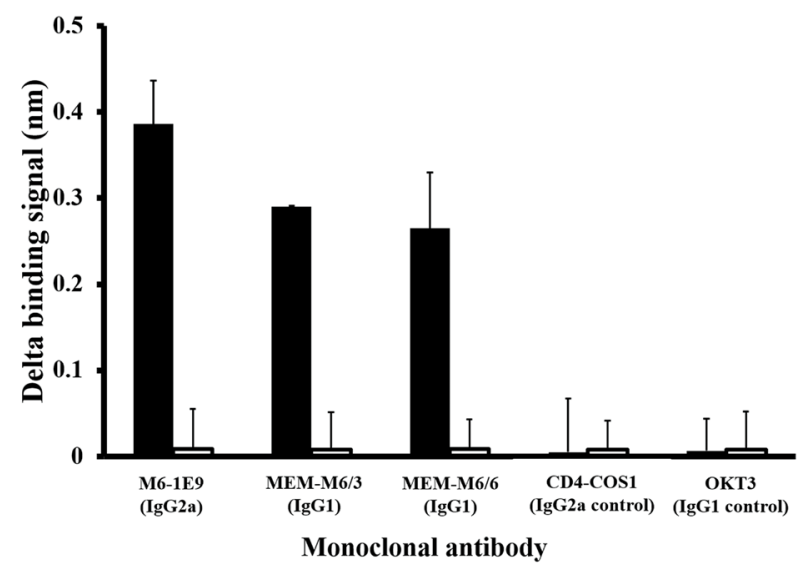

C

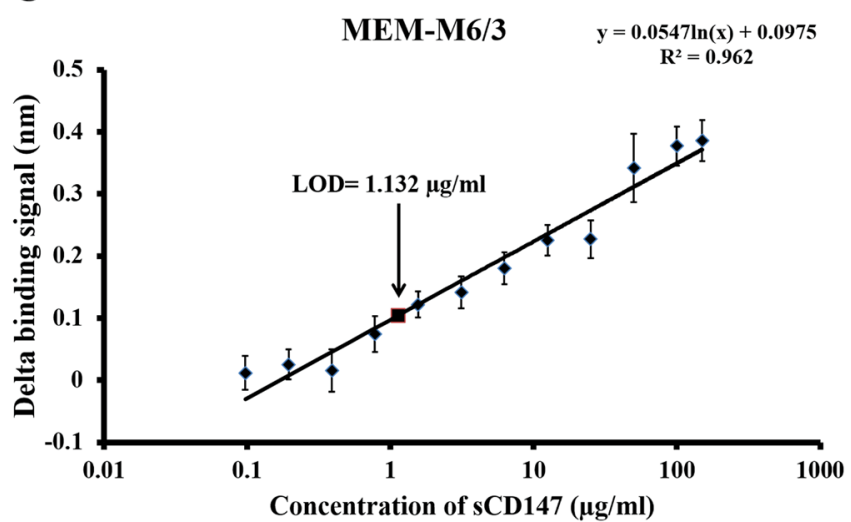

The combination of BLItz and the use of different anti -CD147 mAbs specific for different domains of the CD147 molecule led to the establishment of antibody-based sensors for the detection of different sCD147 forms. When using these sensors in a single step procedure, only the sCD147 general form (containing D1 linked to D2) and the truncated form with the D1 or D2 domain could be determined. For instance, when the sample was positive for the anti-CD147 D1 sensor, only the presence of sCD147 containing D1 and the absence of the truncated form with D2 domain was indicated. The sample positive for the anti-CD147 D2 sensor only indicated the presence of sCD147 containing D2 and the absence of the truncated form with D1 domain. At the same time, the sample positive for both anti-CD147 D1 and anti-CD147 D2 sensors contained, at least, the sCD147 general form. Therefore, the question was raised regarding how to differentiate between the sCD147 general form (D1 linked to D2) and samples containing CD147 D1 and CD147 D2. To address this question, double Ab-biosensors were designed.

B

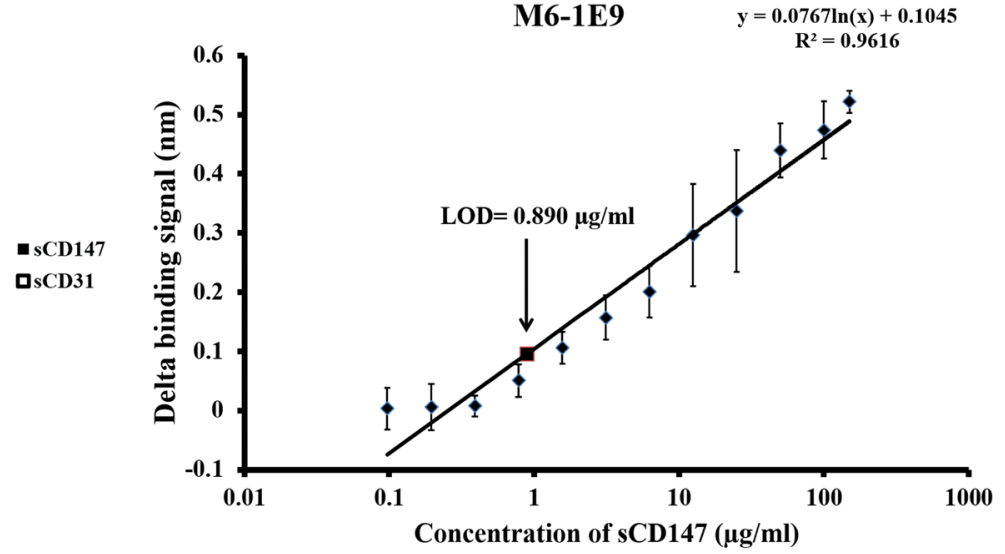

D

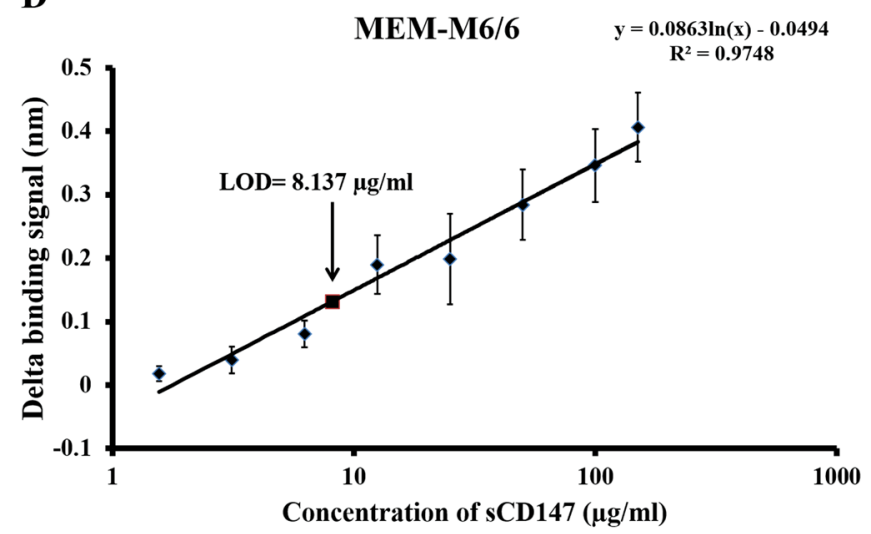

Figure 3. Detection of soluble CD147 by single Ab-biosensor system. The sCD147 general form or sCD31 were loaded into the $\mathrm{Ab}$-sensors and the binding signals were measured using a BLItz biosensor. The delta binding signals of sCD147 and sCD31 to the indicated $\mathrm{mAbs}$ were plotted and presented as the mean $\pm \mathrm{SD}$ of three independent experiments $(\mathrm{A})$. The standard curves were generated to determine sCD147 concentrations using immobilized biotinylated anti-CD147 D1 mAb clone M6-1E9 (B), anti-CD147 D1 mAb clone MEM-M6/3 (C) and anti-CD147 D2 mAb clone MEM-M6/6 (D) with various concentrations of sCD147 general form. The R-squares and the equations are illustrated. The limit of detection (LOD) was calculated as described in Materials and Methods. Mean \pm SD of the data binding signals from three independent experiments; one sensor was tested with one concentration of sCD147 in each experiment, as shown. 
Double Ab-biosensor system for the determination of soluble CD147

By following the same method adopted for the single $\mathrm{Ab}$ -biosensor, a secondary anti-CD147 mAbs specific to either D1 or D2 were added to the system and the reaction was analyzed in real-time by using the BLItz biosensor. This system, called the double Ab-biosensor system, detected sCD147 consisting of D1 linked to D2. The secondary Abs used were non-biotinylated anti-CD147 mAbs, which recognized CD147 domains different to those recognized by the primary biotinylated

A

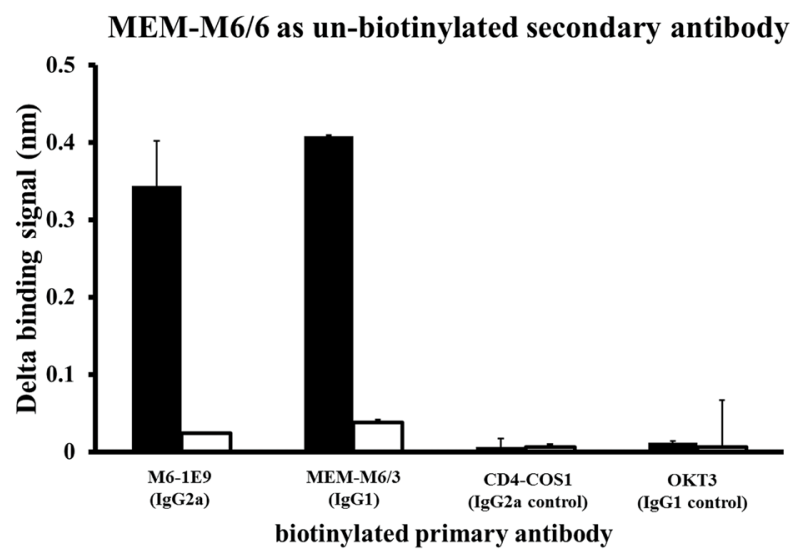

C

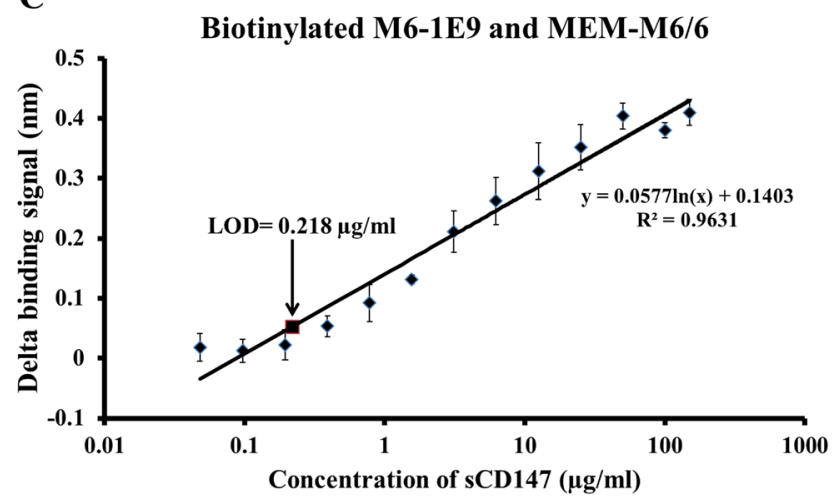

$\mathbf{E}$

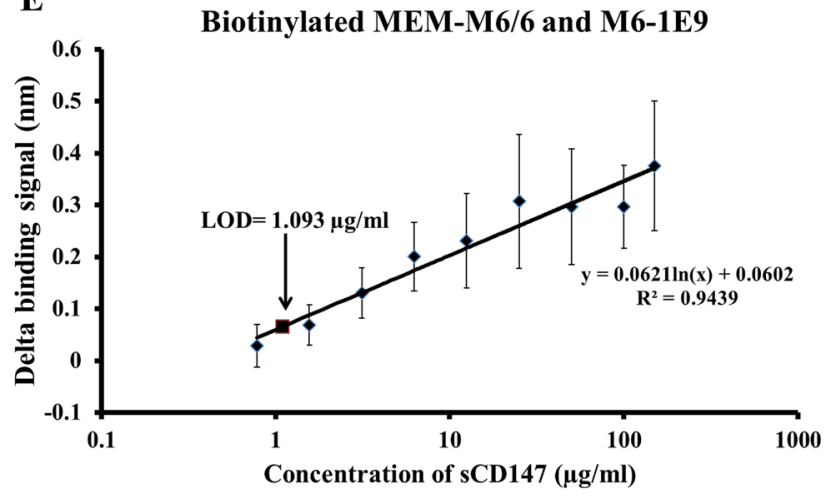

antibody. Using this double Ab-biosensor system, the binding signal of the sCD147 general form (containing D1 linked to D2) to the primary anti-CD147 mAb immobilized on the sensor tip was detected in line with the previous result. As expected, a strong binding signal was observed when the secondary mAbs were added. As the control, the binding signal was undetectable in both irrelevant isotype matched control antibody conditions and $\mathrm{sCD} 31$ protein control conditions (data not shown). Figures $4 \mathrm{~A}$ and $4 \mathrm{~B}$ illustrate the binding signal of the secondary antibody. With this double

B

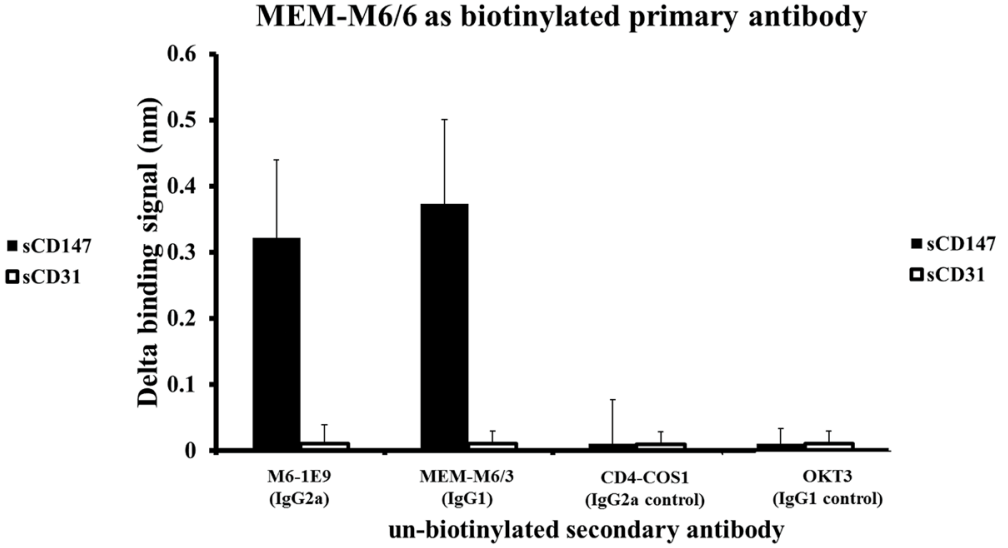

D

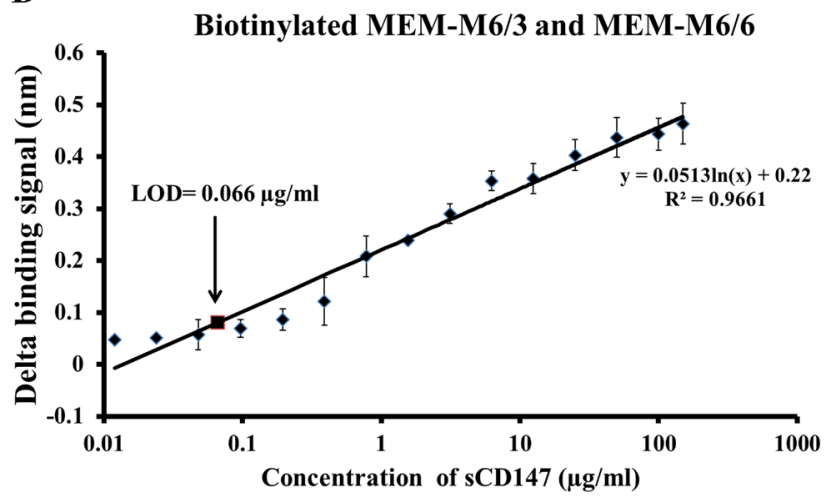

$\mathbf{F}$

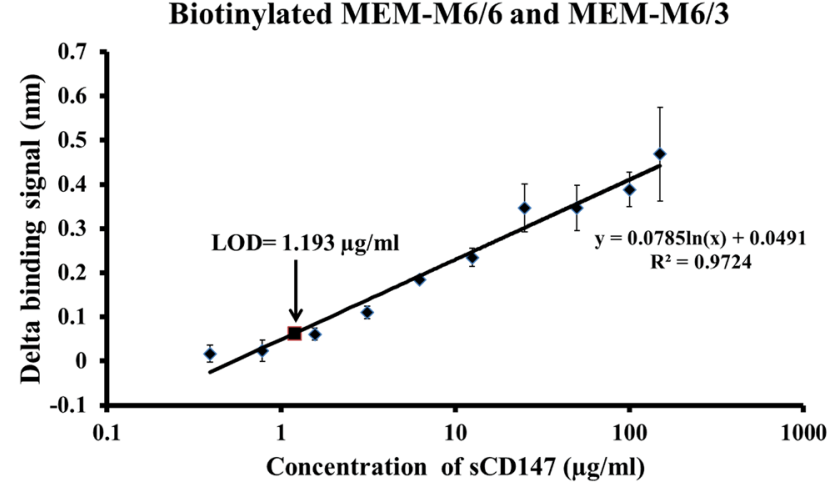

Figure 4. Detection of soluble CD147 by double Ab-biosensor system. After the single Ab-biosensor step, un-biotinylated secondary antibodies were loaded and the binding signals were determined using a BLItz biolayer interferometry biosensor. The delta binding signals of the indicated secondary antibodies to sCD147 or sCD31 on the sensors were plotted and presented as mean \pm SD of three independent experiments (A and B). The standard curves were generated to determine the sCD147 concentrations using the pair of biotinylated and un-biotinylated mAbs: M6-1E9 and MEM-M6/6 (C), MEM-M6/3 and MEM-M6/6 (D), MEM-M6/6 and M6-1E9 (E), and MEM-6/6 and MEM-M6/3 (F) with various concentrations of sCD147 general form. The R-squares and the equations are illustrated. The limit of detection (LOD) was calculated as described in Materials and Methods. Mean \pm SD of the data from three independent experiments; one sensor was tested with one concentration of sCD147 in each experiment, as shown. 

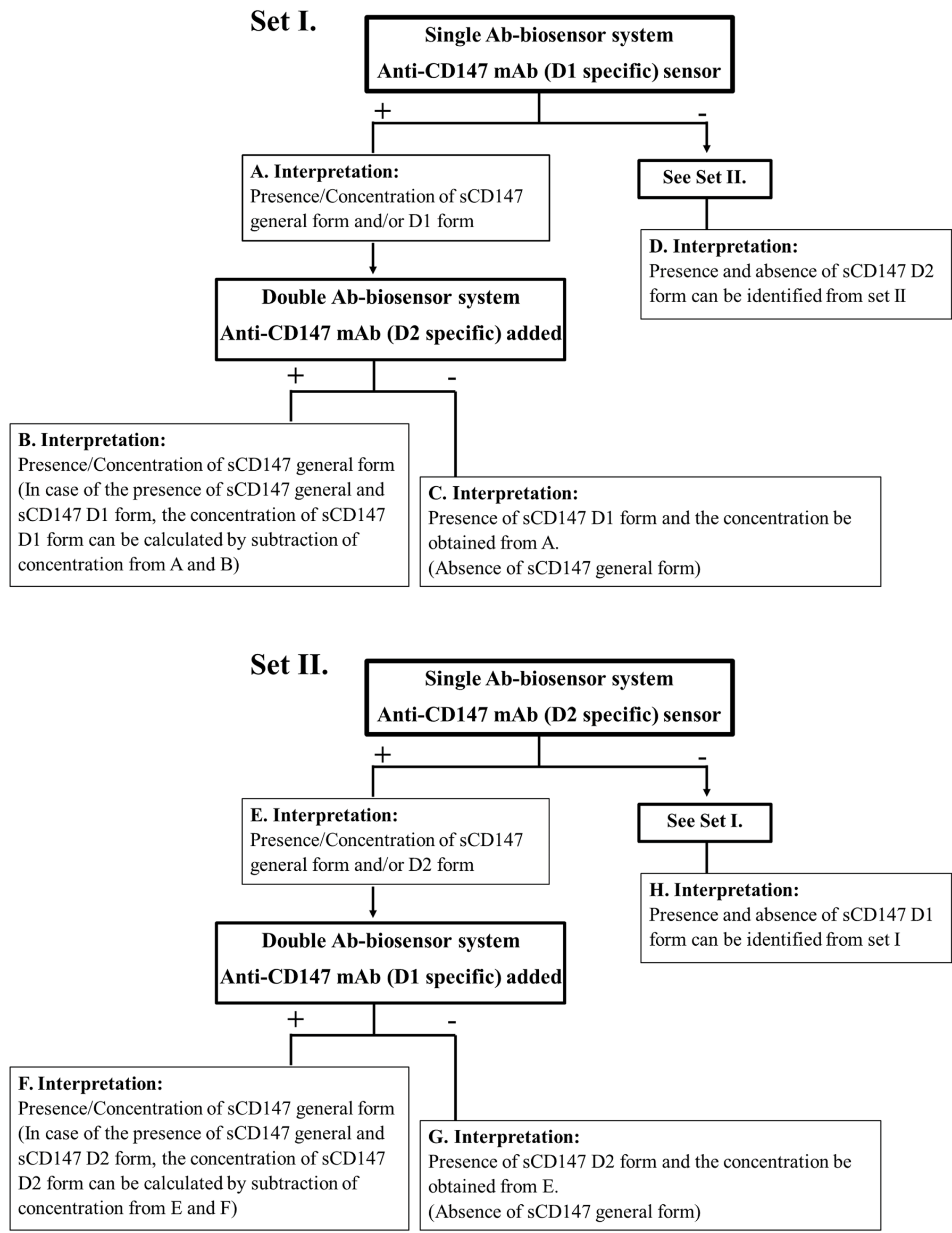

Figure 5. The flowchart demonstrates the strategy and interpretation of using the developed method of single Ab-sensor and double $\mathrm{Ab}$-sensor for the determination of sCD147 isoforms. 
A

Single Ab-biosensor
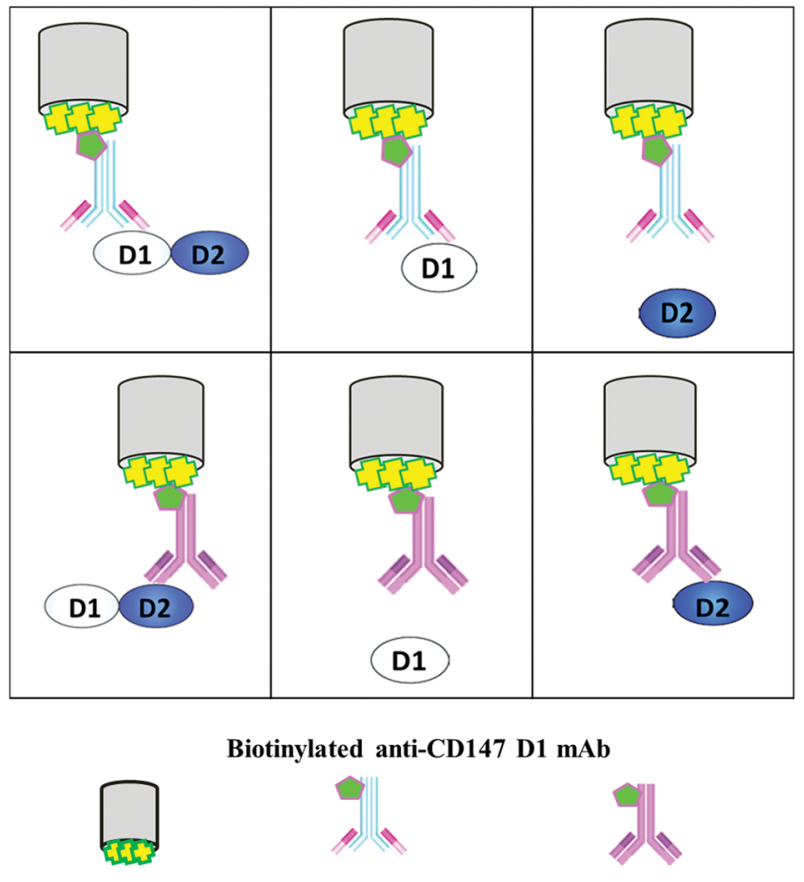

Streptavidin sensor tip

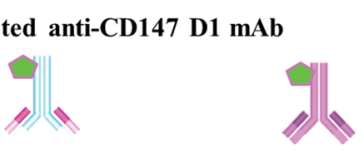

Biotinylated anti-CD147 D2 mAb
B

Double Ab-biosensor

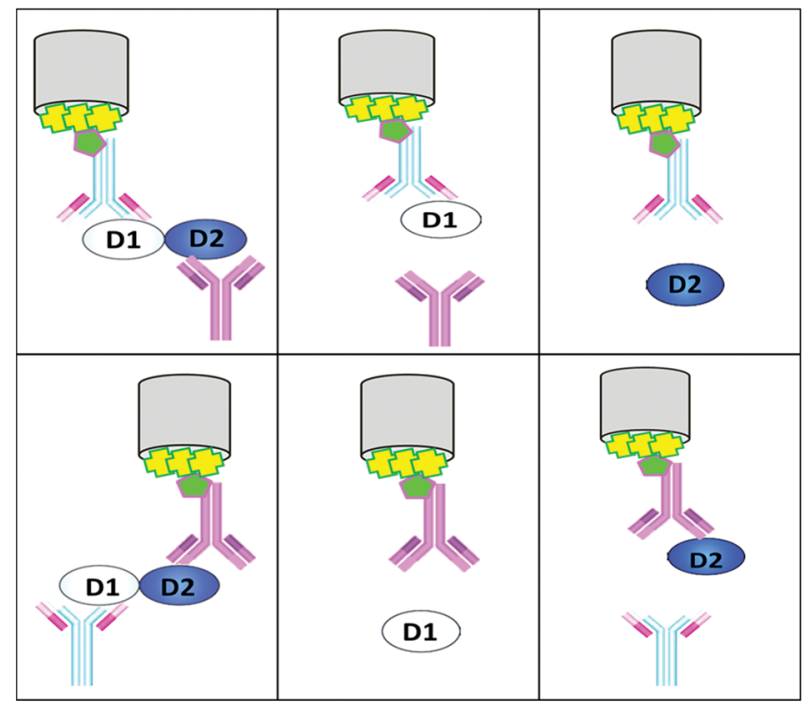

sCD147 D1-D2

SCD147 D2

anti-CD147 D1 mAb

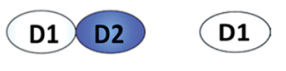

SCD147 D1

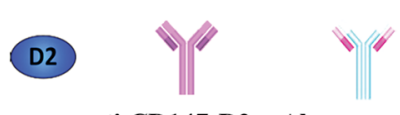

anti-CD147 D2 mAb

Figure 6. Schematic illustration of the model for soluble CD147 isoform detection using Ab-biosensor. (A) Single Ab-biosensor system using biotinylated anti-CD147 mAb specific for CD147 D1 (upper panel) or specific for CD147 D2 (lower panel) immobilized on streptavidin sensors. This system can detect sCD147 general form (D1 linked to D2) and sCD147 D1 or sCD147 D2 isoform. (B) Double Ab-biosensor system for the detection of sCD147 general form (D1 linked to D2). After obtaining a positive binding signal from the single Ab-biosensor assay, secondary anti-CD147 mAbs specific for CD147 D2 or CD147 D1 were added. The association and the dissociation signals of the single Ab-biosensor and the double Ab-biosensor assays were monitored in real-time by using a BLItz biolayer interferometry biosensor.

Ab-sensor strategy, only sCD147 containing D1 linked to D2 was detected.

To determine the optimal conditions, the pairs of antibodies were matched for use as primary and secondary antibodies. Upon using the anti-CD147 D1 clone M6-1E9 mAb as the primary antibody and the anti-CD147 D2 mAb clone MEM-M6/6 as the secondary antibody, it was observed that the LOD was at a concentration of $0.218 \mu \mathrm{g} / \mathrm{mL}$ (Figure 4C). However, the lowest detection limit was obtained at a concentration of $0.066 \mu \mathrm{g} / \mathrm{mL}$ by using the anti-CD147 D1 clone MEM-M6/3, as shown in Figure 4D. Using the anti -CD147 D2 mAb clone MEM-M6/6 as the primary antibody, the LODs obtained were $1.093 \mu \mathrm{g} / \mathrm{mL}$ and $1.193 \mu \mathrm{g} / \mathrm{mL}$, respectively, when the M6-1E9 and the MEM-M6/3 mAbs were used as the secondary antibodies (Figures $4 \mathrm{E}$ and F).

By combining the results of the single Ab-biosensor and the double Ab-biosensor, the presence and concentrations of the general sCD147 isoform (D1 linked to D2) or the truncated forms (D1 or D2) could therefore be determined, as shown in Figure 5.

\section{Discussion}

CD147 is a multifunctional leukocyte surface molecule involved in many cellular functions..$^{710,33}$ Expression of this molecule is associated with cancer progression and metastasis. ${ }^{11-15}$ In addition to the membrane-bound form, sCD147 has been detected in body fluids in several diseases. ${ }^{12,23-26}$ sCD147 containing either two Ig domains (general form) or only the N-terminal Ig domain (D1 form) has been identified in different diseases. ${ }^{16-19,26,29}$ Detection of sCD147 in clinical samples could be a valuable marker for disease diagnosis and prognosis. It has also been reported that different extracellular domains of CD147 have distinct binding partners and functions. ${ }^{21}$ Understanding and identifying the structure of shed CD147 might lead to better knowledge of cellular mechanisms and systems biology.

Due to the current limitations of the available sCD147 detection methods, the structure of shed sCD147 is still not well-characterized. ${ }^{17-19,29}$ To address this problem, in the present study we developed an $\mathrm{Ab}$ based-biosensor system by using BLItz. Biolayer interferometry is a type of optical interferometry. ${ }^{34,35}$ The BLItz instrument shines white light down the biosensor and collects the light reflected back. The interference patterns of white light reflected from two surfaces, i.e. an internal reference layer and a layer of molecules bound to its partner on the biosensor tip, are analyzed. The binding of molecules onto the biosensor tip results in a change in interference, which is converted to a binding signal in 
real-time monitoring. The binding signals are correlated with the concentration and size of the analytical molecules. ${ }^{34,35}$ Using the combination of BLItz and different anti-CD147 mAbs specific for different extracellular domains of the CD147 molecule, $\mathrm{Ab}$ based-biosensors were established to rapidly and simultaneously measure and characterize sCD147 isoforms.

In order to test for the presence of different isoforms of sCD147, two types of Ab-biosensors, namely the single Ab-biosensor and the double Ab-biosensor, were designed (Figure 6). For the single Ab-biosensor, anti-CD147 mAb specific for CD147 D1 or D2 was coated onto sensor tips and used for the quantification of sCD147 using the BLItz optical interferometric biosensor in real-time (Figure 6). The single Ab-biosensor could detect the presence of both the general sCD147 form (having D1 linked to D2) and sCD147 D1 or sCD147 D2 isoforms in the tested samples, depending on the $\mathrm{Ab}$ used. For the double Ab-biosensor, following the single Ab-biosensor assay, secondary anti-CD147 mAbs specific for CD147 D2 or CD147 D1, respectively, were added and monitored in real-time using a BLItz biosensor (Figure 6). By combining the results obtained from the single Ab-biosensor and the double Ab-biosensor, the presence and concentrations of the general sCD147 isoform (D1 linked to D2) or truncated forms (D1 or D2) could be determined (Figure 5). This system for the determination of sCD147 characteristics has never been described before. The developed method is novel and of interest for uncovering new information regarding sCD147 isoforms.

With the developed $\mathrm{Ab}$ sensors, the optical interferometric sensing system directly monitors the molecular binding of sCD147 to the Ab sensor. Once the molecules bind to the surface of the biosensor, the binding signal is immediately sent out and, consequently, the presence of sCD147 can be determined. This makes the established method capable of completing the detection and identification of sCD147 isoforms in real time within 10 minutes. In order to employ the developed system for measuring sCD147 in real clinical samples, the cross-reactivity of all clones of the anti-CD147 $\mathrm{mAbs}$ to other proteins in serum were evaluated. The results demonstrate that no cross-reactivity of the anti-CD147 mAbs to other serum proteins was observed (data not shown). The generated system is, therefore, suitable for the clinical laboratory measurement of sCD147 for disease diagnosis and prognosis. In addition, as the developed method can be used for the detection of sCD147 isoforms, it might lead to better understanding of cell biology, body abnormalities and the pathology of disease. Furthermore, the concept of this method can be applied to detect other molecules of interest.

In summary, in the current study, we established an $\mathrm{Ab}$ biosensor system for the detection and characterization of the shedding forms of CD147. The developed Ab-sensors are very simple to operate: the sensor tips only have to be dipped into samples and sCD147 can be detected in real-time. By using different anti-CD147 mAbs, the distinct form of sCD147 can be determined. The developed system could be a valuable tool for the diagnosis and prognosis of CD147-associated diseases. As sCD147 isoforms are still contentious, the developed system may be employed for the detection of sCD147 isoforms, which will lead to a better understanding of CD147 shedding. In addition, this may increase our knowledge of cellular mechanisms and their associations with abnormalities and diseases of the human body.

\section{Acknowledgments}

This study was supported by the Research Grant of the Faculty of Associated Medical Sciences, Chiang Mai University, the Thailand Research Fund (grant number TRG5780017), and the TRF Senior Research Scholar (RTA5980007). Nuchjira Takheaw is a doctoral candidate of the Royal Golden Jubilee Ph.D. program of the Thailand Research Fund (PHD/0121/2557). Saichit Khummuang is a doctoral candidate in the Royal Golden Jubilee Ph.D. program of the Thailand Research Fund and Chiang Mai University (PHD/0064/2555).

\section{Competing interests}

The authors declare that they have no competing interests.

\section{References}

1. Kasinrerk W, Fiebiger E, Stefanova I, Baumruker T, Knapp W, Stockinger H. Human leukocyte activation antigen M6, a member of the Ig superfamily, is the species homologue of rat OX-47, mouse basigin, and chicken HT7 molecule. J Immunol. 1992;149:847-54.

2. Miyauchi T, Kanekura T, Yamaoka A, Ozawa M, Miyazawa S, Muramatsu T. Basigin, a new, broadly distributed member of the immunoglobulin superfamily, has strong homology with both the immunoglobulin V domain and the beta-chain of major histocompatibility complex class II antigen. J Biochem. 1990;107:316-23.

3. Biswas C, Zhang Y, DeCastro R, Guo H, Nakamura T, Kataoka H, et al. The human tumor cell-derived collagenase stimulatory factor (renamed EMMPRIN) is a member of the immunoglobulin superfamily. Cancer Res. 1995;55:434-9.

4. Stockinger H, Ebel T, Hansmann C, Koch C, Majdic O, Prager E, et al. CD147 (neurothelin/basigin) Workshop Panel Report. In: Kishimoto T, Kikutani H, Von Dem Borne AEGK, Goyert SM, Mason DY, Miyasaka M, Moretta L, Okumura K, Shaw S, Springer TA, Sugamura K, Zola H, editors. Leucocyte Typing VI. New York: Garland Publishing. 1997. p. 760-5.

5. Muramatsu T. Basigin: a multifunctional membrane protein with an emerging role in infections by malaria parasites. Expert Opin Ther Targets. 2012;16:999-1011.

6. Belton RJ, Jr., Chen L, Mesquita FS, Nowak RA. Basigin-2 is a cell surface receptor for soluble basigin ligand. J Biol Chem. 2008;283:17805-14.

7. Kasinrerk W, Tokrasinwit N, Phunpae P. CD147 monoclonal antibodies induce homotypic cell aggregation of monocytic cell line U937 via LFA-1/ ICAM-1 pathway. Immunology. 1999;96:184-92.

8. Khunkeawla P, Moonsom S, Staffler G, Kongtawelert P, Kasinrerk W. Engagement of CD147 molecule-induced cell aggregation through the activation of protein kinases and reorganization of the cytoskeleton. Immunobiology. 2001;203:659-69.

9. Chiampanichayakul S, Peng-in P, Khunkaewla P, Stockinger H, Kasinrerk W. CD147 contains different bioactive epitopes involving the regulation of cell adhesion and lymphocyte activation. Immunobiology. 2006;211: 167-78.

10. Yurchenko V, Constant S, Eisenmesser E, Bukrinsky M. Cyclophilin-CD147 interactions: a new target for anti-inflammatory therapeutics. Clin Exp Immunol. 2010;160:305-17.

11. Weidle UH, Scheuer W, Eggle D, Klostermann S, Stockinger H. Cancer -related issues of CD147. Cancer Genomics Proteomics. 2010;7:157-69.

12. Xiong L, Edwards CK, Zhou L. The biological function and clinical utilization of CD147 in human diseases: a review of the current scientific literature. Int J Mol Sci. 2014;15:17411-41.

13. Muraoka K, Nabeshima K, Murayama T, Biswas C, Koono M. Enhanced expression of a tumor-cell-derived collagenase-stimulatory factor in urothelial carcinoma: its usefulness as a tumor marker for bladder cancers. Int J Cancer. 1993;55:19-26.

14. Polette M, Gilles C, Marchand V, Lorenzato M, Toole B, Tournier JM, et al. Tumor collagenase stimulatory factor (TCSF) expression and localization in human lung and breast cancers. J Histochem Cytochem. 1997;45:703-9. 
15. Zhang Q, Zhou J, Ku XM, Chen XG, Zhang L, Xu J, et al. Expression of CD147 as a significantly unfavorable prognostic factor in hepatocellular carcinoma. Eur J Cancer Prev. 2007;16:196-202.

16. Sidhu SS, Mengistab AT, Tauscher AN, LaVail J, Basbaum C. The microvesicle as a vehicle for EMMPRIN in tumor-stromal interactions. Oncogene. 2004;23:956-63.

17. Egawa N, Koshikawa N, Tomari T, Nabeshima K, Isobe T, Seiki M. Membrane type 1 matrix metalloproteinase (MT1-MMP/MMP-14) cleaves and releases a $22-\mathrm{kDa}$ extracellular matrix metalloproteinase inducer (EMMPRIN) fragment from tumor cells. J Biol Chem. 2006;281:37576-85.

18. Taylor PM, Woodfield RJ, Hodgkin MN, Pettitt TR, Martin A, Kerr DJ, et al. Breast cancer cell-derived EMMPRIN stimulates fibroblast MMP2 release through a phospholipase $\mathrm{A}(2)$ and 5-lipoxygenase catalyzed pathway. Oncogene. 2002;21:5765-72.

19. Haug C, Lenz C, Diaz F, Bachem MG. Oxidized low-density lipoproteins stimulate extracellular matrix metalloproteinase Inducer (EMMPRIN) release by coronary smooth muscle cells. Arterioscler Thromb Vasc Biol. 2004;24:1823-9.

20. Belton RJJ, Chen L, Mesquita FS, Nowak RA. Basigin-2 is a cell surface receptor for soluble basigin ligand. J Biol Chem. 2008;283:17805-14.

21. Bai Y, Huang W, Ma LT, Jiang JL, Chen ZN. Importance of N-glycosylation on CD147 for its biological functions. Int J Mol Sci. 2014;15:6356-77.

22. Tang W, Chang SB, Hemler ME. Links between CD147 function, glycosylation, and caveolin-1. Mol Biol Cell. 2004;15:4043-50.

23. Yanaba K, Asano Y, Tada Y, Sugaya M, Kadono T, Hamaguchi Y, et al. Increased serum soluble CD147 levels in patients with systemic sclerosis: association with scleroderma renal crisis. Clin Rheumatol. 2012;31:835-9.

24. Traian CM, Liliana MV, Denisa P, Leontina MB, Crina S, Cristiana M. Abnormalities in soluble CD147 / MMPs / TIMPs axis in ankylosing spondylitis patients with and without a history of acute anterior uveitis. Rev Romana Med Lab. 2014;22:479-96.

25. Pennings GJ, Yong AS, Wong C, Al-Tamimi M, Gardiner EE, Andrews RK, et al. Circulating levels of soluble EMMPRIN (CD147) correlate with levels of soluble glycoprotein VI in human plasma. Platelets. 2014;25: $639-42$
26. Wu J, Hao ZW, Zhao YX, Yang XM, Tang H, Zhang X, et al. Full-length soluble CD147 promotes MMP-2 expression and is a potential serological marker in detection of hepatocellular carcinoma. J Transl Med. 2014;12:190.

27. Moonsom S, Tayapiwatana C, Wongkham S, Kongtawelert P, Kasinrerk W. A Competitive ELISA for quantifying serum CD147: reduction of soluble CD147 levels in cancer patient sera. Hybridoma (Larchmt). 2010;29:45-52.

28. Zheng D, Zhu X, Ding X, Yin Y, Li G. Sensitive detection of CD147/ EMMPRIN and its expression on cancer cells with electrochemical technique. Talanta. 2013;105:187-91.

29. Tang Y, Kesavan P, Nakada MT, Yan L. Tumor-stroma interaction: positive feedback regulation of extracellular matrix metalloproteinase inducer (EMMPRIN) expression and matrix metalloproteinase-dependent generation of soluble EMMPRIN. Mol Cancer Res. 2004;2:73-80.

30. Pata S, Tayapiwatana C, Kasinrerk W. Three different immunogen preparation strategies for production of $\mathrm{CD} 4$ monoclonal antibodies. Hybridoma (Larchmt). 2009;28:159-65.

31. Koch C, Staffler G, Huttinger R, Hilgert I, Prager E, Cerny J, et al. T cell activation-associated epitopes of CD147 in regulation of the $\mathrm{T}$ cell response, and their definition by antibody affinity and antigen density. Int Immunol. 1999;11:777-86.

32. Prager E, Sunder-Plassmann R, Hansmann C, Koch C, Holter W, Knapp W, et al. Interaction of CD31 with a heterophilic counterreceptor involved in downregulation of human T cell responses. J Exp Med. 1996;184:41-50.

33. Ruiz S, Castro-Castro A, Bustelo XR. CD147 inhibits the nuclear factor of activated T-cells by impairing Vav1 and Racl downstream signaling. J Biol Chem. 2008;283:5554-66.

34. ForteBio.com [Internet]. California: Pall corporation; c2016 [cited 2016 Sep 29]. BLI Technology; [about 1 screen]. Available from: http://www.fortebio. com/bli-technology.html.

35. ForteBio.com [Internet]. California: Pall corporation; c2016 [cited 2016 Sep 29]. BioLayer Interferometry (BLI) - How Does it Work?; [about 1 screen]. Available from: http://www.fortebio.com/interactions/Spring_2012 /page5.html. 